\title{
Multimedia Training Material Development to Facilitate the Learning of Plant Systematics
}

Romel Palomino Jaramillo, Eng. ${ }^{1}$, Andrea Rojas Jiménez, Eng. ${ }^{2}$, Óscar Agudelo Varela, Msc. ${ }^{3}$, Miguel Bonilla Morales, Msc.

Universidad de los Llanos ${ }^{1},{ }^{3},{ }^{4}$, , Colombia ${ }^{1},{ }^{3},{ }_{4}$, romel.palomino@unillanos.edu.co, andrea.rojas@ unillanos.edu.co, oscar.agudelo@unillanos.edu.co,mmbonillam@unal.edu.co

\begin{abstract}
In the present study information for the development of multimedia teaching materials Taxonomy and Biogeography of Passiflora (supersection Tacsonia section Colombiana), for the course of Plant Systematic Biology Program at the Universidad de los Llanos is collected, showing how you can conduct a scalable development usable, useful and intuitive, through strategies based on the user, through the development of he stages of project planning, design, coding, testing and delivery. To show this strategy has resulted, an interactive type software application web that has points interactivity in content and some activities at the end, along with an ebook that collects all the information relating to subject treated. Upon completion, the outlook in the short, medium and long term management of educational resources, leaving one structure for the realization of new developments applied to different courses within the University arises.

Keywords-- Plant Systematic Biology, development of multimedia teaching materials, Taxonomy and Biogeography of Passiflora.
\end{abstract}

Digital Object Identifier

(DOI):http://dx.doi.org/10.18687/LACCEI2016.1.1.131

ISBN: 978-0-9822896-9-3

ISSN: 2414-6390

$14^{\text {th }}$ LACCEI International Multi-Conference for Engineering, Education, and Technology: "Engineering Innovations for Global Sustainability", 20-22 July 2016, San José, Costa Rica. 


\title{
Multimedia Training Material Development to Facilitate the Learning of Plant Systematics
}

\author{
Romel Palomino Jaramillo, Eng. ${ }^{1}$, Andrea Rojas Jiménez, Eng. ${ }^{2}$, Óscar Agudelo Varela, Msc. ${ }^{3}$, Miguel Bonilla \\ Morales, Msc. ${ }^{4}$ \\ Universidad de los Llanos ${ }^{1,2,3,4}$,Colombia ${ }^{1,2,3,4}$,romel.palomino@unillanos.edu.co,andrea.rojas@unillanos.edu.co, \\ oscar.agudelo@unillanos.edu.co,mmbonillam@unal.edu.co
}

\begin{abstract}
In the present study information for the development of multimedia teaching materials Taxonomy and Biogeography of Passiflora (supersection Tacsonia section Colombiana), for the course of Plant Systematic Biology Program at the Universidad de los Llanos is collected, showing how you can conduct a scalable development usable, useful and intuitive, through strategies based on the user, through the development of the stages of project planning, design, coding, testing and delivery.

To show this strategy has resulted, an interactive type software application web that has points interactivity in content and some activities at the end, along with an ebook that collects all the information relating to subject treated.

Upon completion, the outlook in the short, medium and long term management of educational resources, leaving one structure for the realization of new developments applied to different courses within the University arises.
\end{abstract}

Keywords-- Plant Systematic Biology, development of multimedia teaching materials, Taxonomy and Biogeography of Passiflora.

\section{INTRODUCCIÓN}

En la actualidad, aumentan los esfuerzos de las Tecnologías de Información y Comunicación (TIC) por incursionar en el ámbito de la educación y el aprendizaje orientado a los estudiantes de educación superior, de tal forma que facilite los procesos de enseñanza-aprendizaje y así crear un escenario educativo donde exista una apropiación de contenidos para lograr una estructura analítica y de autoaprendizaje, que se vea reflejada en la recursividad, modernización y creatividad en los estudiantes y la comunidad académica en general.

La integración de las TIC en la educación y procesos de formación permite que comunidades académicas puedan acceder a una amplia gama de recursos educativos en la web [1]; por lo tanto, es realmente importante conocer la existencia de dichos recursos digitales que han sido diseñados para que docentes y estudiantes logren mejorar los procesos de enseñanza y aprendizaje; pero más importante aún, es que los mismos estudiantes que se encuentran estrechamente relacionados con las TIC, colaboren con el crecimiento de estos recursos desarrollando contenidos interactivos multimedia en diversos campos para establecer un incremento exponencial de la dinámica enseñanza-aprendizaje.

Digital Object Identifier (DOI): http://dx.doi.org/10.18687/LACCEI2016.1.1.131 ISBN: 978-0-9822896-9-3

ISSN: 2414-6390
Son muchos los retos que impone este nuevo modelo educativo basado en el acceso al conocimiento para entornos de aprendizaje apoyados en TIC. Uno de ellos es, como se menciona anteriormente, lograr involucrar a los estudiantes en trabajos colaborativos con profesores y expertos para lograr crear entornos óptimos, donde la gestión del contenido implique la introducción de estrategias innovadoras que aprovechen de forma adecuada estas tecnologías.

Por otro lado y continuando con los retos que se imponen, cabe mencionar, que si bien los contenidos educativos han estado presentes en diversos procesos de formación en los ámbitos académicos e institucionales, en Colombia no se ha tenido en cuenta la gestión de dichos contenidos como un tema fundamental de investigación y optimización de recursos humanos y técnicos.

Lo anterior no significa que el tema de contenidos educativos se haya manejado equivocadamente en los ambientes de formación apoyados con TIC, lo que muestra, es que es necesario plantear la problemática en los ambientes académicos, investigativos y de formación docente, ya que el país está viviendo un auge en ambientes virtuales de aprendizaje que apoyen los procesos de enseñanza y aprendizaje, además de la creciente oferta de programas de formación completamente virtuales que también han sido promovidos por el Ministerio de Educación.

Bajo este contexto, el presente documento propone el desarrollo de material didáctico multimedia sobre Taxonomía y Biogeografía de Passiflora (supersección Tacsonia sección Colombiana) para el curso Sistemática Vegetal del programa de Biología de la Universidad de los Llanos, logrando así que estudiantes de Ingeniería de Sistemas de la Universidad, se apropien por participación de recursos educativos de enseñanza-aprendizaje en el ámbito de la Biología, para el caso actual.

\section{DESCRIPCIÓN DEL TRABAJO}

\section{A. Profesionales Implicados}

Para el desarrollo de estos materiales educativos se hace necesario involucrar un equipo de profesionales multidisciplinar que, utilizando un modelo de trabajo en conjunto generaren resultados satisfactorios y de beneficio a

$14^{\text {th }}$ LACCEI International Multi-Conference for Engineering, Education, and Technology: "Engineering Innovations for Global Sustainability”, 20-22 July 2016, San José, Costa Rica. 
la comunidad educativa. Este modelo de trabajo permite aportar conocimientos, destrezas y conductas mediante grupos de discusión e intercambios de información [2].

Las personas en el equipo de trabajo se conforma de acuerdo a diferentes áreas profesionales; gestión, pedagogía, diseño gráfico, desarrollo hipermedia y expertos en Biología vegetal, procedentes del Grupo de Investigación Horizonte Mediático, ver fig. 1 .

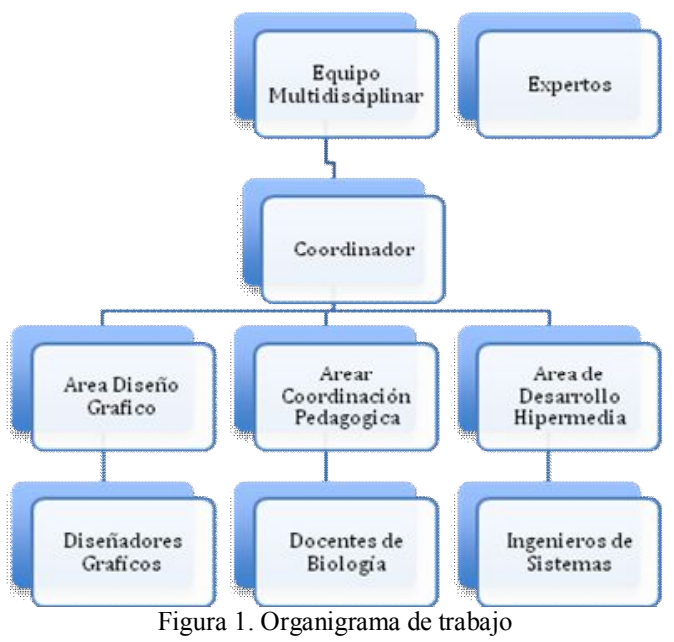

\section{B. Flujo de trabajo}

El flujo de trabajo se divide en cinco fases: equipo multidisciplinar, coordinación, desarrollo pedagógico, diseño y desarrollo hipermedia. La comunicación entre las entapas se realiza a través de diversos métodos de comunicación, tanto síncrona como asíncrona, entre los que destacan las historias de usuario que explican las tareas a realizar en la siguiente fase, ver Fig. 2, en el cual se ilustra el flujo de trabajo para realizar el Material Didáctico Multimedia (MDM).

\section{Equipo Multidisciplinar}

Se encarga de reunir los distintos puntos de vista de los profesionales, discutirlos y plantear soluciones acorde a la dificultad plateada, por otra parte, su trabajo resulta en seguir un proceso de evaluación continua de los productos obtenidos por con el objetivo de introducir sucesivas mejoras y detectar lo antes posible los futuros fallos e impedir así que se propaguen a una siguientes fase.

\section{Coordinación}

Es la responsable de acompañar, liderar y administrar el proceso del desarrollo del material didáctico multimedia de acuerdo a los objetivos planteados por el equipo multidisciplinar y las historias de usuario resultantes de las otras fases. También es la encargada de fomentar la comunicación entre las diferentes fases.

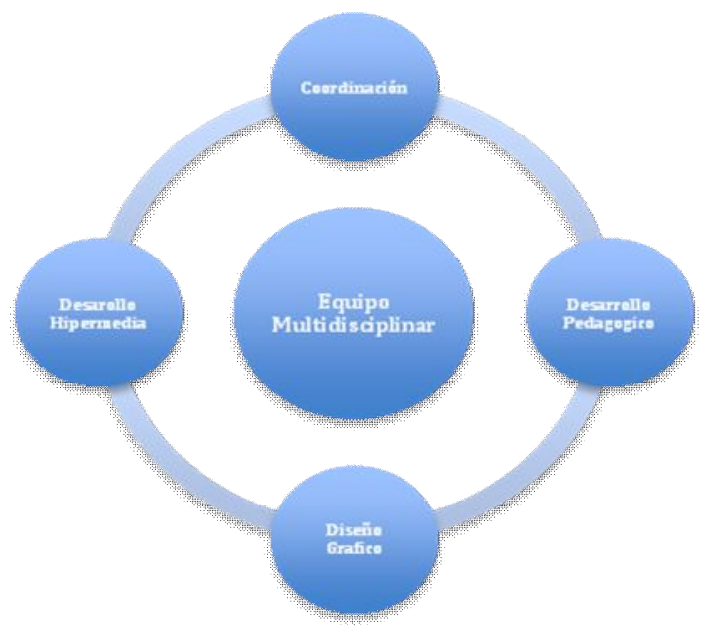

Figura 2. Diagrama de flujo de trabajo MDM

\section{Desarrollo Pedagógico}

Docentes que por su experiencia en la enseñanza en Sistemática Vegetal establecen la estructura de los contenidos e indican las ilustraciones a tener en cuenta en el diseño del Material Didáctico Multimedia.

\section{Diseño gráfico}

De acuerdo a indicaciones suministradas por el equipo multidisciplinar son los encargados de realizar los Mockups, dar la forma visual definitiva tanto al interfaz como a los contenidos, teniendo en cuenta consideraciones estéticas y ergonómicas. Esto incluye la selección de tipografias, colores, y las ilustraciones.

\section{Desarrollo hypermedia}

La función del equipo hipermedia consiste en construir la estructura lógica del Material Didáctico Multimedia, y a partir de ella completar el contenido suministrado por los docentes en la fase de desarrollo pedagógico, teniendo en cuenta los diseños aportados por los diseñadores gráficos.

\section{- Metodología de desarrollo hypermedia}

Este desarrollo contempla la elaboración de dos MDM, uno es un ebook y el otro es un MDM para ambiente Web, se procede a su construcción mediante elementos de la metodología ágil eXtreme Programming (desarrollo iterativo e incremental, Programación en parejas, cercanía con el experto del tema); ya que las metodologías ágiles son

$14^{\text {th }}$ LACCEI International Multi-Conference for Engineering, Education, and Technology: "Engineering Innovations for Global Sustainability”, 20-22 July 2016, San José, Costa Rica. 
adecuadas para el desarrollo de aplicaciones web interactivas [3].

\section{- Aplicación de HTML 5}

La herramienta para el desarrollo hipermedia de los MDM en la última década fue delegada a Adobe Flash. Debido a su línea de tiempo, que permitía realizar animaciones vistosas y agradables [4].

La llegada de HTML5, en compañía de todo lo que su nombre expone, generó que el desarrollo en Adobe Flash perdiera su importancia [5], ya que las animaciones generadas en compañía de HTML5, JavaScript y CSS3 [6] eran suficientemente buenas para derrocar esta fuerte herramienta de desarrollo.

\section{- Migración sustancial}

Los cambios abruptos, no son lo más recomendado para la migración de una tecnología y más, cuando este cambio deja atrás una década de conocimientos y de forma de hacer las cosas.

Por este motivo se seleccionó Adobe Animate; una herramienta que gracias a su línea de tiempo y símbolos nos recuerdan mucho al clásico Adobe Flash, pero sus etiqueta, desarrollo con lenguaje JavaScript y exportación en HTML dan como resultado un enlace entre un conocimiento previo y el avance de una nueva tecnología.

\section{- Iteraciones}

Estas iteraciones se realizan cuantas veces sean necesarias y sean oportunas en consenso con el equipo interdisciplinar para obtener el Material Didáctico Multimedia (MDM).

Para el desarrollo de Sistemática Vegetal se realizaron 5 iteraciones para obtener el MDM definitivo.

\section{Iteración 1}

En esta iteración, los docentes presentaron la división del proyecto en cuatro capítulos, el grupo de diseño gráfico entregaron los primeros prototipos y el grupo de desarrollo hipermedia seleccionaron las herramientas para la implementación de los prototipos, ver Fig. 3.

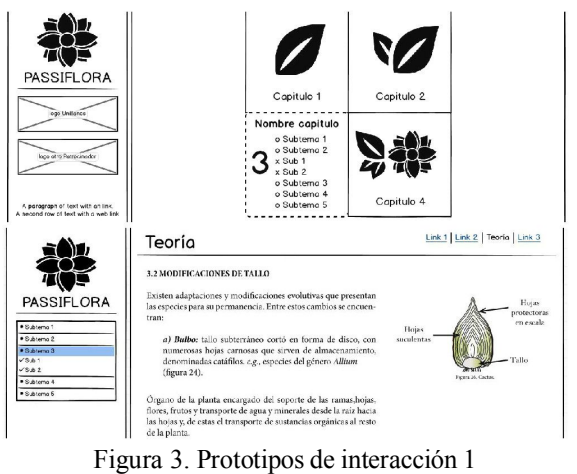

\section{Iteración 2}

En la segunda iteración, los docentes generaron el contenido preliminar para el capítulo uno y dos [7], los diseñadores seleccionaron los colores que utilizaría la aplicación y los desarrolladores multimedia maquetaron la estructura de la misma, ver Fig. 4.

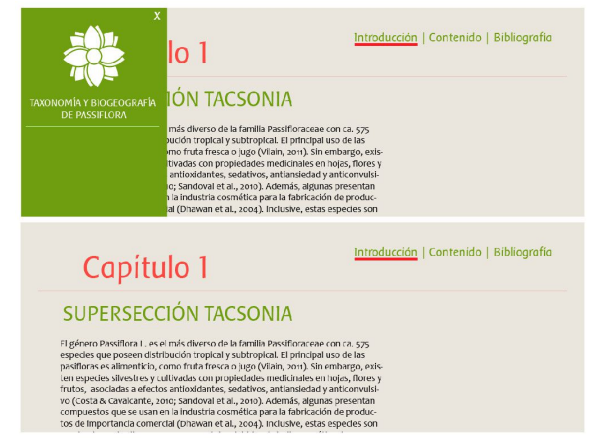

Figura 4. Segunda iteración

Iteración 3

Los docentes generaron el contenido del capítulo 3 y 4 [8], los diseñadores hicieron un cambio de diseño en búsqueda de mayor usabilidad y los desarrolladores maquetaron dicha funcionalidad, ver Fig. 5.

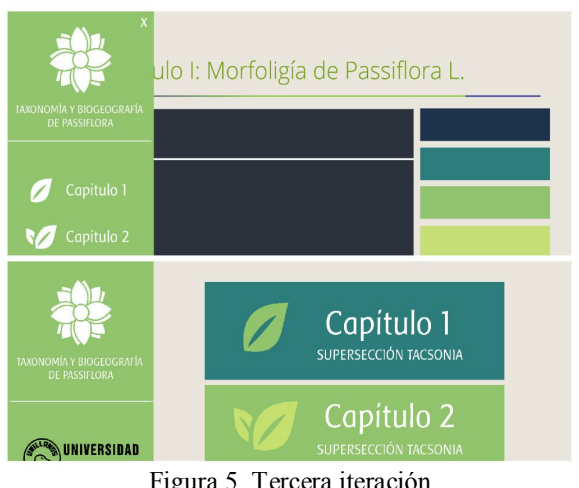

Figura 5. Tercera iteración

$14^{\text {th }}$ LACCEI International Multi-Conference for Engineering, Education, and Technology: "Engineering Innovations for Global Sustainability", 20-22 July 2016, San José, Costa Rica. 


\section{Iteración 4}

En la cuarta iteración los docentes revisaron el contenido, realizando ajustes teniendo en cuenta [9], los diseñadores entregaron ilustraciones y los desarrollares agregaron funcionalidad al material didáctico, ver Fig. 6.

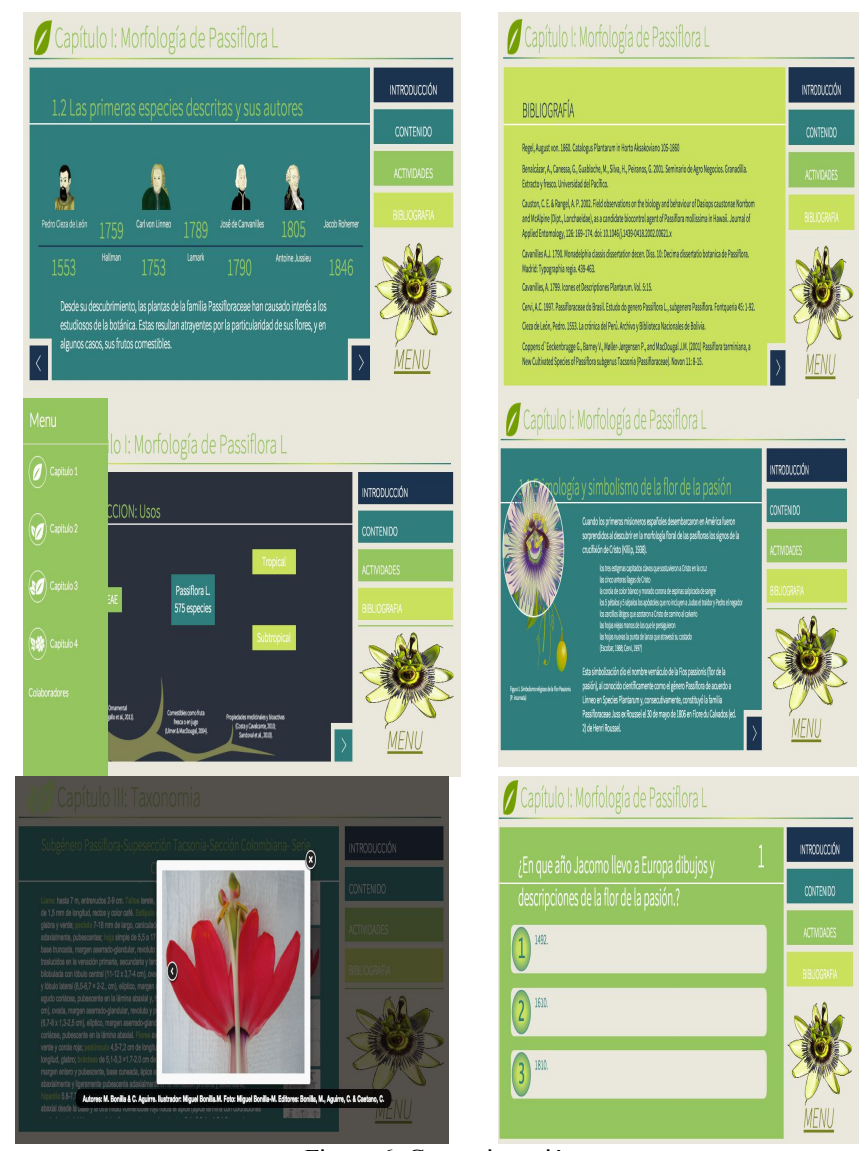

Iteración 5

Figura 6. Cuarta iteración

El coordinador realizo algunos ajustes de usabilidad en la aplicación, cuyas modificaciones implementaron los desarrolladores.

\section{RESULTADOS}

Recurso docente en formato electrónico (e-book) interactivo en taxonomía y biogeografía de Passiflora (supersección Tacsonia sección Colombiana).

Modelo para el desarrollo de material didáctico multimedia para facilitar el aprendizaje en instituciones educativas.

La tabla de contenido contiene hipervínculos que le permite dirigirse a la temática seleccionada.
El ebook está en formato epub y swf para la correcta visualización desde cualquier navegador.

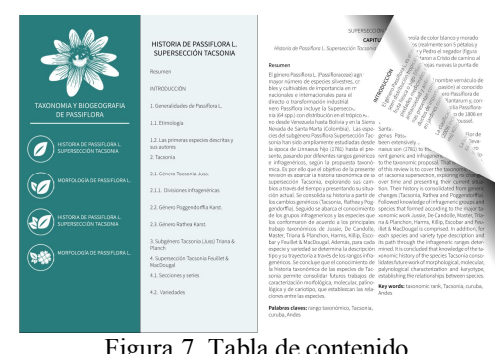

Software interactivo de apoyo educativo orientado a fomentar el aprendizaje de Taxonomía y Biogeografía de Passiflora (supersección Tacsonia sección Colombiana).

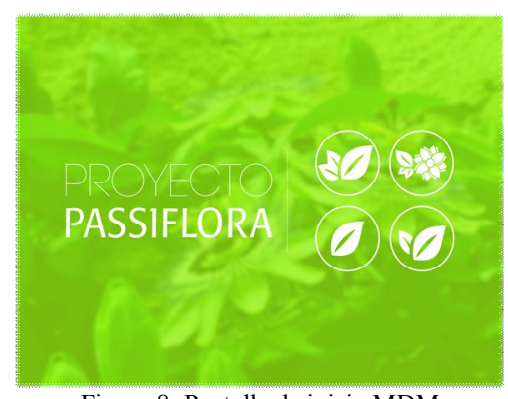

Figura 8. Pantalla de inicio MDM

\section{CONCLUSIONES}

Involucrar un grupo interdisciplinar de profesionales para el desarrollo de un Material Didáctico Multimedia, garantiza un desarrollo de mayor calidad.

Para que este modelo de desarrollo funcione, debe prevalecer la comunicación entre las diferentes fases. De tal manera, el educador (es) y educando puedan regular el proceso de aprendizaje.

Es posible replicar este modelo para el desarrollo de un Material Didáctico Multimedia en diferentes ámbitos de la academia enfocados principalmente a la enseñanza de la taxonomía, una disciplina compleja de aprender en el campo de la biología.

Los educando, mediante la iteración de la tecnología digital fortalecen el aprendizaje en un área específico, generando mejores resultados por la autorregulación y automonitoreo cognitivo que pueden lograr.

$14^{\text {th }}$ LACCEI International Multi-Conference for Engineering, Education, and Technology: "Engineering Innovations for Global Sustainability”, 20-22 July 2016, San José, Costa Rica. 


\section{REFERENCIAS}

[1] Ángel Boza Carreño María de la O, Toscano Cruz, Buenas prácticas en integración de las TIC en educación en Andalucía: Dos estudios de caso Universidad de Huelva, 2011

[2] Martínez, R. A., Pérez, M. H., Sampedro, A., Martínez, Á.: Evaluación y diagnóstico de procesos de formación en entornos virtuales de aprendizaje. En del Moral (coord.): Sociedad del conocimiento, ocio y cultura: un enfoque interdisciplinar. KRK, Oviedo (2004) 81-103

[3] Martínez, R. A., Miláns del Bosch, M., Granda, E., Lupiáñez, F., Pérez, H., Martínez, Á., Sampedro, A.: Aspectos organizativos y didácticos implicados en la elaboración de material educativo multimedia (MEM) para el fomento de la motivación empresarial. Revista Iberoamericana de Educación a Distancia, Vol. 6, Núm. 1, (Junio 2003) 141-157

[4] Priolo, Sebastian. Métodos Agiles Desarrolladores. User. ISBN 9871347979 .

[5] Mauzo, Fabricio y makedonsky, Mariano. Flash: Desarrollo profesional. Tecnicas avanzadas y buenas prácticas. Manuales users. 1a ed. Buenos Aires, Fox Andina Alaga, 2011. ISBN 9871857004. 320p.

[6] Percival, John. HTML5 Advertising. Apressus Series. Expert's voice in Web development. Apress, 2012. ISBN 1430246022. 350 p.

[7] Ocampo Pérez, John; Coppens d'Eeckenbrugge, Geo; Restrepo, María; Jarvis, Andy; Salazar, Mike; Caetano, CreuciDiversity of Colombian Passifloraceae: biogeography and an updated list for conservation. Biota Colombiana . jun 2007.

[8] Alexandra Hernández y Rodrigo Bernal, Lista de Especies de Passifloraceae de Colombia Instituto de Ciencias Naturales, Universidad Nacional de Colombia, 2000.

[9] Carolina Millán J., Patricia Chacón C. Germán Corredor, Development, longevity, and ovipositión of Heliconiuscharithonia (Lepidoptera: Nymphalidae) on two species of Passiflora 2010

$14^{\text {th }}$ LACCEI International Multi-Conference for Engineering, Education, and Technology: "Engineering Innovations for Global Sustainability", 20-22 July 2016, San José, Costa Rica. 